\title{
Comment la physique spatiale est entrée au laboratoire
}

La création du centre d'étude spatiale des rayonnements (toulouse, 1964-1970)

How space physics entered the laboratory: the creation of the Centre d'étude spatiale des rayonnements (Toulouse, 1964-1970)

Jérôme Lamy

\section{OpenEdition Journals}

Édition électronique

URL : https://journals.openedition.org/ress/3998

DOI : $10.4000 /$ ress.3998

ISSN : 1663-4446

Éditeur

Librairie Droz

Édition imprimée

Date de publication : 25 mai 2018

Pagination : 85-111

ISSN : 0048-8046

Référence électronique

Jérôme Lamy, "Comment la physique spatiale est entrée au laboratoire ", Revue européenne des sciences sociales [En ligne], 56-1 | 2018, mis en ligne le 25 mai 2021, consulté le 08 janvier 2022. URL http://journals.openedition.org/ress/3998 ; DOI : https://doi.org/10.4000/ress.3998 


\title{
COMMENT LA PHYSIQUE SPATIALE EST ENTRÉE AU LABORATOIRE LA CRÉATION DU CENTRE D'ÉTUDE SPATIALE DES RAYONNEMENTS (TOULOUSE, 1964-1970)
}

JÉRÔME LAMY Université Toulouse Jean Jaurès, CERTOP jerome.lamy@laposte.net

\begin{abstract}
Résumé. Cet article détaille la façon dont la physique spatiale s'est constituée en discipline à travers la genèse du Centre d'étude spatiale des rayonnements à Toulouse, dans les années 1960. À partir des écologies liées d'Andrew Abbott, il est possible de reconstituer la formation simultanée d'un corpus scientifique et d'un laboratoire. Les affrontements autour du lieu d'implantation signalent la compétition engagée avec la physique nucléaire. Le regroupement de quelques jeunes physiciens autour de problématiques novatrices et d'instrumentations inédites participe de ce mouvement de coagulation disciplinaire. Enfin, les tentatives pour arrimer le laboratoire à d'autres structures, plus ancrées, témoignent de la fragilité du processus en cours.
\end{abstract}

Mots-clés: discipline, écologie, laboratoire, physique spatiale.

\begin{abstract}
This article details how space physics was formed into a discipline through the genesis of the Centre d'étude spatiale des rayonnements, in Toulouse during the 1960's. Using Andrew Abbott's linked ecologies, it is possible to reconstruct the simultaneous formation of a discipline and of a laboratory. Clashes around the site of the laboratory reveal the competititon involved with nuclear physics. The gathering of some young physicists around innovative problems and instrumentation construct this disciplinary movement. Finally, attemps to associate the laboratory with more solidly established structures show the fragility of the process.
\end{abstract}

Keywords: discipline, ecology, laboratory, space physics. 
Le second conflit mondial a profondément transformé la manière de faire de la physique. L'incorporation de cette discipline dans le processus de guerre (notamment via le projet Manhattan à Los Alamos) a modifié non seulement ses fondements théoriques (et tout particulièrement dans la compréhension des particules élémentaires), mais également ses modes d’organisation. La massification des laboratoires, la croissance des instruments (Galison et Hevly, 1992), la «culture de l'urgence» (Pestre, 2004, p. I2), ainsi que le management par projet (Lamy, 2or ia) ont reconfiguré cette discipline.

Parallèlement, et en lien avec ce nouveau dimensionnement, la physique connaît une transformation profonde avec une segmentation intra-disciplinaire particulièrement poussée. Cette segmentation avait déjà commencé entre les deux conflits mondiaux ${ }^{1}$. Toutefois, après 1945, elle s’est accompagnée d’une spécialisation instrumentale de plus en plus poussée. Ainsi, des instruments spécifiques deviennent les points d’appuis essentiels pour certaines sous-disciplines de la physique. C'est le cas, par exemple, de la chambre à bulle pour la physique des particules, comme l’a mis en évidence Peter Galison (1997, p. 3 I3-42 I). Il existe, malgré tout, des circulations artefactuelles rendues possibles par la généricité de certains instruments (Shinn et Ragouet, 2005, p. 17 I-178).

L'émergence des technologies spatiales - dans le contexte de la guerre froide - s'organise autour de la construction de nouveaux instruments spécifiquement adaptés aux conditions de recueil des données aux limites et hors de l'atmosphère. Dans la grande division intra-disciplinaire qui travaille la physique de la seconde moitié du $\mathrm{xx}^{\mathrm{e}}$ siècle, l'avènement des technologies spatiales fournit une série d’opportunités scientifiques pour mieux comprendre les phénomènes physiques de l'atmosphère, l'évolution de la végétation (Mack, 1990) mais aussi le climat, la météorologie (Kirge, 2000), le fonctionnement des océans (Conway, 2006) ainsi que l'ensemble des objets traditionnellement étudiés par l'astrophysique. Les ballons, les sondes puis les satellites permettent de s'abstraire d’un certain nombre de contraintes liées aux perturbations atmosphériques. En

I Par exemple la physique des états solides et la physique quantique se structurent très fortement dans l'entre-deux guerres, voir Hoddeson, Bran, Teichmann, Weart, 1992; Forman, 1971. 
s'appuyant sur une technologie dédiée, spécialement conçue pour recueillir des données physiques spécifiques, les scientifiques du domaine spatial inventent, peu à peu, à partir de la fin des années 1950, leurs propres méthodes, défendent leur spécialité, délimitent leur territoire cognitif et tentent de se structurer institutionnellement.

Cet article, qui s'inscrit dans le champ de la sociologie des sciences, entend précisément comprendre comment la physique spatiale entre au laboratoire en prenant pour point d’appui l'exemple de la création du Centre d'étude spatiale des rayonnements (CESR) à Toulouse dans les années 1960. Il sagit de saisir la façon dont des physiciens des particules, plutôt centrés sur les questions classiques de leur sous-discipline, ont fait le choix d'une réorientation de leurs pratiques en direction de la physique spatiale. La question des choix de spécialités est centrale en sociologie des sciences : il s'agit de comprendre comment les éléments cognitifs, techniques, instrumentaux, mais aussi politiques sarticulent pour densifier un segment spécifique de recherche (Mullins, 1972; Edge, Mulkay, 1976).

Le CESR ne recouvre pas à lui tout seul la physique spatiale française; il n'est qu’un laboratoire parmi d'autre orienté vers cette spécialité. Mais en concentrant notre attention sur un espace scientifique restreint et ancré, nous pouvons mieux discerner le travail politique, scientifique et technique mené par une poignée d'acteurs pour qu'existe à Toulouse un laboratoire consacré à la physique spatiale. Il s'agit en quelque sorte de proposer l'histoire processuelle d'une spécialité, sans l'abstraire des grands mouvements de déploiement dans lesquels elle est prise à partir des années 1960 avec le développement des technologies spatiales, mais en retraçant le travail élémentaire de défrichage épistémique, politique, scientifique et instrumental que suppose l'édification d'un laboratoire. Comment émergent une spécialité et un laboratoire? Quels sont les mécanismes de constitution d'une écologie de la physique spatiale à Toulouse? L'analyse se focalise sur les premiers moments de ce qui n’est pas encore une écologie, cette période instable où l'inachèvement peut mener à l'échec. 
Pour mener à bien à cette analyse des déplacements intra-disciplinaires, nous aurons recours aux propositions conceptuelles d'Andrew Abbott centrées sur les écologies liées (Abbott, 2005)르. Les écologies sont des segments relativement distincts de la vie sociale au sein desquels des acteurs se reconnaissent entre eux dans une activité spécifique. Si l'on considère les différentes disciplines de la physique dans les années d’après-guerre comme des écologies en formation, il est possible d’analyser leurs rapprochements, leurs frottements ou leurs séparations comme des processus historiques d'autonomisation. Abbott précise que les écologies évoluent dans un environnement compétitif qui oblige ceux qui œuvrent à leur maintien à défendre ou à étendre leur champ de compétence; elles ne sont cependant pas systématiquement en compétition, des liens particuliers et contingents peuvent relier des aires d’action initialement disjointes. L’analyse du processus de création du CESR en termes d'écologies permet de comprendre comment s'opère ce travail social de structuration des disciplines caractérisé par des jeux subtiles de rapprochements institutionnels et de séparations cognitives. Surtout, en nous focalisant sur ce moment particulier de création d'un laboratoire, il est possible de saisir sur le vif la gamme large des appuis cognitifs, techniques, politiques et institutionnels qui sous-tendent l'émergence d'une discipline.

L’enquête que nous avons menée repose sur un dépouillement d’archives inédites conservées au sein du CESR ${ }^{3}$ et d'entretiens réalisés avec quelques-uns de ses membres. Si les sources orales sont précieuses dans la reconstitution des premières heures du laboratoire, elles peuvent également constituer, de fait, les enquêtés en nomothète parlant pour et au nom de l'institution en réduisant ainsi à un seul point de vue les multiples voies d'accès à l'histoire d'une institution (Star et Griesemer, 1989, p. 309). Le risque est ici d'autant plus grand qu'Henri Reme, ancien directeur du laboratoire, est le seul témoin des débuts du laboratoire.

2 Pour une présentation générale du travail d'Andrew Abbott, voir Fabiani, 2003; Demazière et Jouvenet, 2016. Arnaud Saint-Martin a proposé une approche écologique de l'astronomie française à la Belle Époquequi examine les efforts d'alignements professionnels (modes de recrutement, hiérarchie des tâches) à la quête d'un monopole cognitif autour des instruments et des pratiques (Saint-Martin, 2008).

3 Ces archives sont en cours de classement et de transfert aux Archives départementales de Haute-Garonne. 
Nous disposons, en outre, d'un entretien réalisé par Michel Grossetti en I99I du fondateur du CESR, Francis Cambou, décédé en 2004 . L'abondance relative des archives écrites permet d'obvier à une histoire «officielle» du laboratoire. La littérature grise (rapports d’activités, projets inaboutis, plans d’organisation, correspondance interne et comptes rendus de débats au sein des instances universitaires) fournissent un matériau particulièrement dense, pour qu'il soit possible d'éviter une épopée in verba magistri.

L’article décline les trois éléments constituant le CESR en tant qu'écologie : je dégagerai d'abord les conditions concrètes d'installation du laboratoire en retraçant le double travail de démarcation épistémique et immobilier; je présenterai ensuite la constitution du petit groupe de chercheurs autour d'un ensemble plus ou moins stabilisé d'instruments et de pratiques; enfin, je décomposerai le travail de jonction mené par les membres du CESR en de multiples directions pour assurer leur pérennité institutionnelle et scientifique.

\section{LE LABORATOIRE COMME CHAMP DE BATAILLE}

Le «tournant géographie» récent dans les études sociales des sciences (Naylor, 2005) a mis en exergue l'importance de la matérialité dans les espaces de recherche. Le lieu, conçu non seulement comme «arène » social, mais également comme un espace matériel et contraint permet une stabilisation de l'activité scientifique: il forme un cadre prédéfini pour que se déploient des interactions sociales entre chercheurs, ensuite, il possède une certaine plasticité qui ne l'enferme pas dans les intentions premières des architectes, enfin et malgré ces propriétés évolutives, le lieu de science impose une certaine capacité de structuration interne qui interdit des opérations non prévues (Gieryn, 2002). Afin de reconstituer la chaîne politique et scientifique qui relie le CESR comme lieu à son existence institutionnelle, je reconstitue brièvement l'environnement social qui accompagne sa création. 
Dans les années 1960, le général de Gaulle poursuit une politique d’indépendance nationale qui s'articule à un ensemble de projets technoscientifiques d’ampleur comme le nucléaire ou le spatial. Le Centre national d'études spatiales (CNES) est officiellement créé en I96I : il s’agit d’une agence de moyens chargée de favoriser le développement de la recherche spatiale en proposant des ressources techniques et managériales à un nombre limité de laboratoires scientifiques prenant l'espace pour objet. Parallèlement à cette ébullition institutionnelle, le gouvernement entreprend un vaste mouvement de décentralisation scientifique et choisit d'y impliquer le CNES. Le Centre technique spatial doit se reconstituer à Toulouse de la fin des années 1960 au début des années 1970. La ville accueille également des écoles spécialisées : l'École nationale supérieure d’aéronautique et l'École nationale d’aviation civile (Lamy, 20ı Ia). C’est dans ce contexte, qui noue les questions d’aménagement du territoire et de politique d'indépendance nationale, que le CESR est créé à Toulouse. Lassemblée de la Faculté des sciences de Toulouse prend l'initiative, le 9 mars 1964, de valider le projet du jeune professeur Francis Cambou qui avait suggéré l'établissement, autour de lui, d'un Institut d'études spatiales des rayonnements ${ }^{5}$. Arrivé à Toulouse en 196i à la demande du physicien Daniel Blanc pour mettre sur pied un Centre de physique nucléaire, Cambou se rend rapidement compte que cette spécialité ne serait jamais développée dans la région Midi-Pyrénées. Il envisage de poursuivre ses travaux en «physique nucléaire fondamentale » à Paris, mais reste attentif au processus de décentralisation du CNES. Il a formé un petit groupe d'étudiants et se résout finalement à persévérer à Toulouse dans le domaine de la physique spatiale. Je reviendrai plus loin sur ce moment de conversion du capital scientifique, mais auparavant je souhaite mettre en exergue l'importance des questions matérielles (et notamment immobilières) dans la nouvelle distribution du campus toulousain. Le prix intellectuel à payer pour qu'existe un laboratoire dédié aux rayonnements spatiaux est celui d'une rupture avec Daniel Blanc. Jusqu'en 1964, les deux hommes travaillaient au sein du Laboratoire de physique nucléaire, l'émergence de la nouvelle entité ne 
s'accompagne pas immédiatement de l'obtention de nouveaux locaux. Cambou dispose de quelques pièces «étroites, encombrées de matériel scientifique dans l'ancienne Faculté des sciences de Toulouse et n’a de cesse de dénoncer la situation «dramatique» dans laquelle se trouve son équipe ${ }^{6}$. En attendant de disposer d’un laboratoire en propre, il mène une bataille acharnée pour obtenir quelques concessions territoriales de la part du Laboratoire de physique nucléaire?

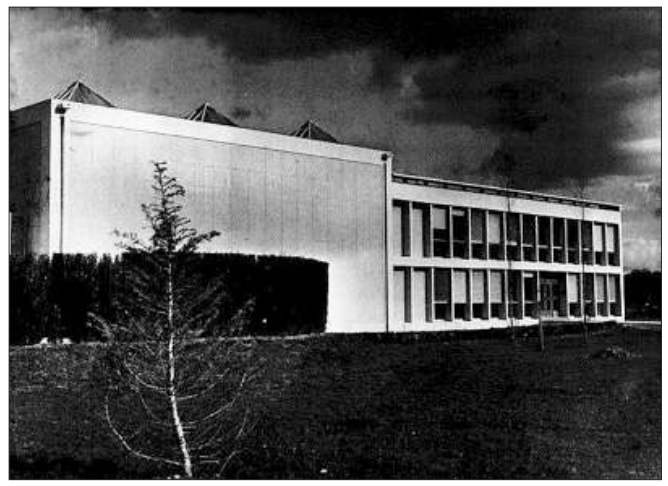

Bâtiment de recherches de physique atomique, Le Centre de physique atomique et nucléaire, Rapport du professeur Blanc, 1968-1968.

L'acmé de cette lutte quasi-géostratégique se déroule lors d'une séance de la Commission de physique de la Faculté des sciences en novembre 1966. Ni Blanc, ni Cambou ne sont présents, mais ils ont fait part de leurs demandes respectives aux intervenants. Cambou fait préciser qu'il attend la décision finale qui lui permettrait d'obtenir des locaux neufs sur le campus et indique que, d'ici là, il souhaite disposer «d'un étage [...] du bâtiment de physique ». Blanc assure quant à lui qu'il ne «lui est pas possible de céder des pièces » pour développer son service. Finalement, la Commission de physique donne raison à Cambou et lui attribue ıoo à I20 $\mathrm{m} 2$ dans les nouveaux locaux du

6 Ibid.

7 ACESR, «Lettre de Francis Cambou au professeur Coucher, président de la Commission de physique», 17 octobre 1966. 
campus $^{8}$. Dès son retour, Cambou prend connaissance du compte rendu de la séance; courroucé par les petites résistances à ses prétentions immobilières et malgré l'obtention de locaux supplémentaires, il rédige une missive sans appel à Emma Laudet, attachée d'intendance à la Faculté des sciences. Il indique que si le soutien du Doyen n'est pas plus ferme, il est «prêt à procéder à des arrêts de programmes scientifiques, qui se traduisent ipso facto par la mise à pied d'une dizaine de personnes ». Cambou menace en outre de rendre public ce qu'il qualifie d'«impuissance de l'université »`. La dramatisation des enjeux, l'ancrage des antagonismes et la lutte autour d'un territoire qui ne peut plus être partagé maintiennent ouverte la fracture entre le Laboratoire de physique nucléaire et le CESR. Il s'agit pour Cambou de tracer une frontière matérielle (et immobilière) séparant deux options épistémiques (la physique nucléaire vs la physique spatiale) désormais concurrentes. Toutefois, cette guérilla de bâtiments ne permet pas un ancrage stable du CESR : il reste à conquérir une véritable autonomie territoriale qui suppose des appuis extérieurs à Toulouse. C'est en effet au niveau de l'élaboration du $V^{e}$ Plan que s'organise l'aménagement de la carte scientifique française. Mais, en 1968, le CESR n'est pas retenu par la Commission de la recherche scientifique et technique «pour une construction autonome» dans le futur plan ${ }^{10}$. Cambou cherche alors des appuis financiers auprès d'autres structures. Il écrit par exemple en 1968 au directeur de l'Institut national d'astronomie et de géophysique, Roger Cayrel, pour obtenir quelques subsides. Mais celui-ci lui rappelle que les coupes budgétaires touchent aussi son établissement et qu'il ne peut faire suite à cette demande ${ }^{11}$. Finalement, le ministre de l'Éducation nationale dégage, en novembre 1968, des crédits prévus dans le Ve Plan pour le

8 ACESR, «Compte rendu de la séance du 25 octobre 1966», Commission de physique, Faculté des sciences, université de Toulouse.

9 ACESR, « Lettre de Francis Cambou à Emma Laudet », s.d. [1968].

I0 ACESR, «Lettre du ministre de l'Éducation nationale au directeur général du CNRS», 30 septembre 1968.

II ACESR, «Lettre du directeur de I'Institut national d'astronomie et de géophysique», Roger Cayrel, 13 décembre 1968. 
Service d'aéronomie et les transferts pour la construction du CESR ${ }^{12}$. Le laboratoire comprend donc, dans cette première tranche de travaux, un bloc regroupant les espaces de recherches à proprement parler ainsi qu’un bloc «intégration » pour la mise au point des fusées-sondes et des satellites ${ }^{13}$. Rédigeant, à la fin de l'année 1968, une note pour le recteur d'Académie, Cambou conclut son propos par une longue liste de remerciements qui rend visible les acteurs mobilisés pour assurer le financement des bâtiments du CESR : le directeur de l’Enseignement supérieur, le président, le directeur général du CNRS, le préfet de Région, le maire de Toulouse, et les doyens successifs de la Faculté des sciences ${ }^{14}$. Cette liste matérialise les appuis extérieurs et non réductibles à la seule communauté scientifique locale : la constitution et la consolidation d'un lieu propre à l'écologie de la physique spatiale toulousaine organise un travail réticulaire en direction des instances scientifiques de gouvernement (CNES, CNRS, Enseignement supérieur) ainsi qu'un jeu scalaire entre responsables locaux (les doyens) et nationaux (le ministre de l'Éducation nationale).

La dispute du lieu origine une séparation physique des acteurs, une rupture locative franche. Il ne s'agit pas seulement de pointer les effets de conflits intellectuels qui peuvent être à l'origine de bifurcations de carrières ou de redéploiement disciplinaire : la lutte est d’abord une lutte des places dans laquelle légitimités territoriale et cognitive se superposent. Le repérage, par Cambou, des questions les plus prometteuses en physique (celles qui concernent le spatial) lui permettent d'envisager un «nichage opportuniste» (opportunistic niching [Shinn, I998, p. 155]) dans un environnement concurrentiel.

12 ACESR, «Lettre du ministre de l'Éducation nationale au préfet de la région Midi-Pyrénées», 23 novembre 1968.

13 ACESR, «Complexe aérospatial de Toulouse-Lespinet, Centre d'étude spatiale des rayonnements», 4 décembre 1968.

14 ACESR, Francis Cambou, «Éléments pour Monsieur le Recteur à propos du Centre d'étude spatiale des rayonnements», décembre 1968. 
L'ancrage d'un laboratoire relève donc de processus complexes et labiles : se manifestant d'abord dans le traçage de frontières consécutif à l'acte de rupture avec le laboratoire de physique nucléaire, la constitution du CESR est menacée par le grand mouvement d'organisation du Ve Plan, avant de bénéficier de soutiens institutionnels circonstanciels. Le lieu de l'écologie de la physique spatiale s'établit donc dans un processus de rassemblement large d’appuis hétérogènes (à la fois politiques et scientifiques, locaux et nationaux), combiné à des dynamiques politiques plus ou moins indépendantes (la décentralisation du CNES, l’action de soutien de la préfecture de Région). Ces opérations inchoatives ne se structurent pas selon un plan prévu; toutefois, leurs associations, leurs mises en série ou les bifurcations auxquelles elles donnent lieu ne sont pas pour autant le pur produit du hasard, en ce sens que les acteurs comme Cambou mènent un travail d’alignement permanent des opportunités tendant précisément à jouer de toutes les ressources à sa disposition et de toutes les échelles d’action disponibles.

\section{UN GROUPE EN FUSION DE PHYSICIENS SPATIAUX}

Pour que l'écologie de la physique spatiale émerge et persiste à Toulouse, le groupe d'acteurs engagé dans le contrôle du laboratoire doit revendiquer le monopole d'un «contrôle légitime» sur leur activité (Abbott, 2003, p. 30). Il s'agit donc de construire un collectif orienté sur un territoire épistémique suffisamment proche de la physique nucléaire (une conversion trop radicale serait peu attractive pour les jeunes chercheurs car trop risquée) mais nettement distinct (pour que l'espace cognitif dégagé puisse être pleinement revendiqué). L’approche écologique offre ici un prisme analytique amalgamant deux perspectives habituellement séparées: d'une part les processus sociaux à l'œuvre dans la constitution d'un collectif et d'autre part les mécanismes menant au contrôle d'un ensemble de pratiques clairement identifiables. 


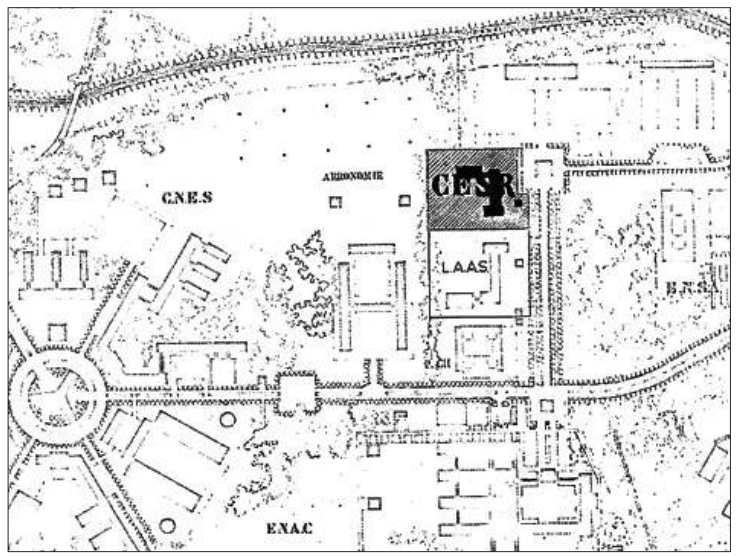

Archives du CESR, Vue d'ensemble du Campus scientifique de Rangueil, ca 1968.

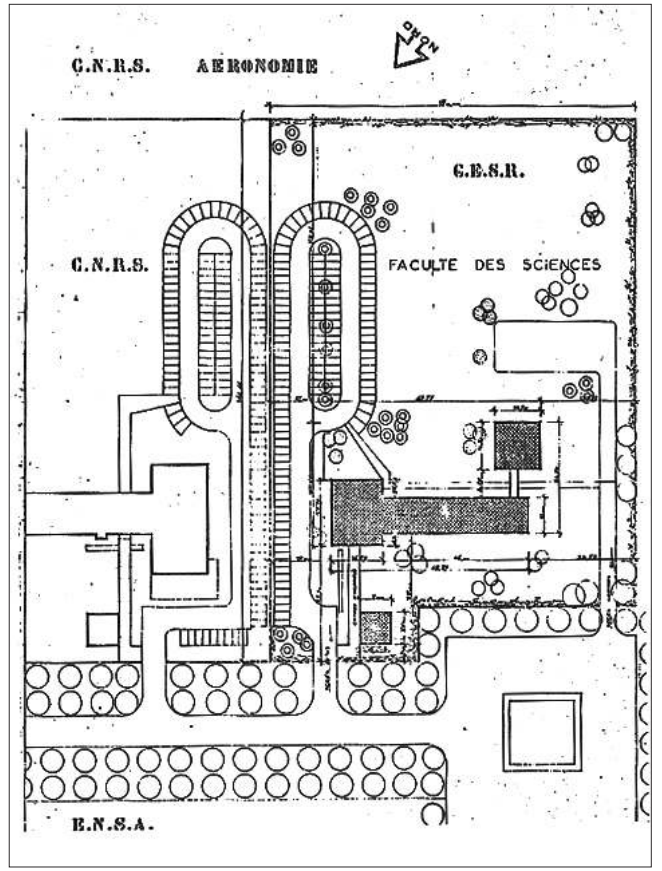

Archives du CESR, Plan du CESR, ca 1968. 
Les divers documents produits par le collectif à l'origine du CESR (e.g. notes, rapports d’activité) témoignent assez de sa propre représentation et de la façon dont il s'envisage dans l'environnement institutionnel et scientifique du campus toulousain. Un historique rédigé en novembre 1968 (probablement par le fondateur Francis Cambou) rappelle que le laboratoire a été créé «en 1964 par le Professeur CAMBOU avec un noyau de "recherche" constitué par des élèves qui travaillaient autour de lui depuis plus de cinq ans $\gg^{15}$. Un mois plus tard, une note anonyme réitère cette idée d'une «petite équipe » autour de Francis Cambou «qui après la physique nucléaire s'était orientée vers la physique spatiale $»^{16}$. Le groupe fondateur apparaît comme un collectif restreint, mais soudé, de conjurés transcendés par la conquête d'un nouveau territoire épistémique (i.e. la physique spatiale). Ce groupe en fusion (Sartre, 1960, p. 424-428) est donc davantage qu'une somme d'individualités ; il articule un projet et un horizon d'attente (i.e. déployer la physique nucléaire à Toulouse) à un ensemble de dispositions matérielles inertes (i.e. le socle de la physique nucléaire, sclérosé dans le contexte toulousain). L'animation du collectif n'est possible qu'à travers le déploiement d'une praxis objectivant l'impasse de la physique nucléaire. Francis Cambou évoquait, dans un entretien réalisé en I99I, l'environnement politico-épistémique dans lequel il arrive à Toulouse :

Je suis venu en [19]6I pour lancer cette équipe de physique nucléaire et très vite on s'est aperçu quau niveau du Plan il n'y aurait jamais de [physique] nucléaire à Toulouse (pas d'investissements lourds). Par conséquent, moi qui voulait faire de la physique nucléaire fondamentale, je devais partir à Paris ou ailleurs car à Toulouse je n'avais aucune chance ${ }^{17}$.

I5 ACESR, Centre d'étude spatiale des rayonnements, novembre 1968.

I6 ACESR, Centre d'étude spatiale des rayonnements, décembre 1968.

17 Entretien de Francis Cambou avec Michel Grossetti, 1991. 
Les premiers élèves qu'il avait formés constituaient un collectif rassemblé autour d'un corpus restreint de thématiques essentiellement lié à la physique nucléaire ou à l'électronique nucléaire ${ }^{18}$. Henri Reme avait ainsi «passé une thèse de troisième cycle en mars [19]63 [...] de spécialité physique des accélérateurs de particules $»^{19}$.

Le passage de la physique nucléaire à la physique spatiale suppose une conversion du capital scientifique et un effort pour changer une trajectoire disciplinairement stable en un déplacement plus risqué. Le régime transitaire, proposé par Terry Shinn et Pascal Ragouet (2005, p. I68-I7I), fournit un cadre d'intelligibilité adéquat pour saisir le passage d'un espace cognitif à un autre. Plus exactement, l'émergence d'opportunités intellectuelles, techniques ou professionnelles motive des franchissements de frontières disciplinaires souvent provisoires. De manière générale, la conversion se fait à moindre coût en opérant un déplacement limité aux régions des savoirs voisins. Shinn et Ragouet insistent sur les trajectoires oscillantes qui, si elles se répètent, peuvent amener la création d'une spécialité ou d'une sous-discipline. Dans le cas du CESR, la trajectoire de Cambou n'est pas oscillante, elle résulte d'une bifurcation nette et sans retour de la physique nucléaire en direction de la physique spatiale; elle s'origine dans un conflit tout à la fois intellectuel (l'obsolescence de la physique nucléaire à Toulouse) et générationnel (i.e. comment se détacher de Daniel Blanc). Shinn et Ragouet restent vagues sur le processus de coagulation qui permet d'atteindre la masse critique suffisante à la fondation d'une nouvelle sous-discipline. Ici, le déplacement de Francis Cambou se double d’une migration de ses élèves. Si la rupture générationnelle dans les processus disciplinaires disruptifs a été très largement documentée par la sociologie des sciences (Mullins, 1972, p.64; Edge et Mulkay, 1976; Ben-David et Collins, I966, p.460), elle reste encore imprécise sur les effets d’opportunité qui la motive. La manière dont Henri Reme, l'un des premiers étudiants de Cambou, évoque son maître rattache l'engagement des disciples à la domination charismatique de Weber qui repose sur l'adhésion totale à une «inspiration» ou à une «volonté concrète de transformation» (Weber, I995 [I92I], p. 323).

18 Ibid.

19 Entretien d'Henri Reme avec Jérôme Lamy, I8 janvier 201 I. 
Henri Reme évoque en termes choisis son adhésion au projet de Cambou: «Quand il s'agit de choisir un sujet de thèse d’État, ça a été le spatial [...]. On a foncé là-dedans avec enthousiasme [...]. C'était nouveau, c'était l'application de nos techniques de laboratoire à l'espace, c'était formidable. $\gg^{20}$

Le CESR présente la spécificité d’associer de jeunes doctorants (notamment Henri Reme, Francis Cotin, Gilbert Vedrennes) à un jeune chercheur (Francis Cambou) dans son processus de formation. Il n'est pas ici question d'une conversion forcée de chercheurs âgés en marge d'une discipline établie (Ben-David et Collins, I966, p.46I-463), mais on assiste au contraire à une rupture générationnelle dans laquelle les nouveaux entrants découpent euxmêmes le territoire cognitif (et matériel) qu'ils comptent s'attribuer.

La proximité épistémique de la physique spatiale avec la physique nucléaire est forte; c'est d’ailleurs ce qui a facilité le mouvement de translation de Cambou. Il reconnaissait en 199I :

petit à petit j'ai orienté toute cette activité vers le spatial, en utilisant dans le spatial la technologie nucléaire. Le spatial, ajoutait-il, consiste à faire des mesures en infrarouge, en ultraviolet, à faire de l'astronomie, des mesures sur optique ou alors on peut utiliser des techniques nucléaires. Donc on a pris notre background de techniques nucléaires pour aborder les mesures de particules dans l'espace et pour regarder en astrophysique d'autres énergies. ${ }^{21}$

Trois des quatre grandes thématiques autour desquelles s'organise le CESR à la fin de l'année 1968 prennent pour objet les particules nucléaires : l'étude des phénomènes magnétosphériques analyse les particules aurorales et la «répartition des particules dans la magnétosphère », le secteur de la «physique cosmique » s'attache à l'examen des rayons $\gamma$ primaires de grandes énergies, enfin, un troisième segment de recherche rassemble les travaux menés sur «l'interaction des particules nucléaires avec les semi-conducteurs», afin de mieux comprendre le comportement des instruments en milieu spatial ${ }^{22}$.

20 Ibid.

21 Entretien de Francis Cambou avec Michel Grossetti, 1991.

22 ACESR, Francis Cambou, «Éléments pour Monsieur le Recteur à propos du Centre d'étude spatiale des rayonnements», décembre 1968. 
L'arsenal technique déployé par les chercheurs et les ingénieurs du CESR est essentiellement constitué de spectromètres dont la plasticité disciplinaire est déjà très largement éprouvée dans les années i960. Ils constituent des instruments génériques, c'est-à-dire des outils construits hors de toute emprise disciplinaire spécifique et ainsi capable d'être investis dans une gamme très variée de recherche. Chaque aire cognitive intéressée décline le concept-souche en un instrument adapté et agencé à ses propres catégories d’analyses (Shinn et Ragouet, 2005, p. I7I-I78 ; Shinn, I997; Joerges et Shinn, I991 ; Lamy et Davoust, 2009). Le spectromètre constitue, depuis le début du $\mathrm{xx}^{\mathrm{e}}$ siècle, l'instrument générique archétypal présent dans les laboratoires de chimie, les observatoires astronomiques et salles d'expérience des physiciens (Bigg, 200I). Les phénomènes auroraux sont repérés grâce à un «détecteur omnidirectionnel permettant une spectrométrie fine du rayonnement $\mathrm{X}$ » ainsi qu'un « détecteur directif destiné à la mise en évidence des déplacements des sources de rayon $\mathrm{X} »^{23}$. Les expériences prévues à l'intérieur du satellite franco-soviétique Roseau porte sur «la spectrométrie des particules de basses énergies dans les différentes zones du milieu spatial $\gg^{24}$. Le programme de travail prévu pour les années 1964 à I966 comprend une «spectrométrie des protons cosmiques » requérant «des compteurs à scintillations munis de cristaux organiques ou minéraux et équipés de circuits discriminateurs de forme $\gg^{25}$. Certaines expériences visant à analyser les effets des particules nucléaires sur les semi-conducteurs sont menées au CERN et à Saclay avec un autre instrument générique de la physique nucléaire, le synchrotron ${ }^{26}$. La physique nucléaire dispose, depuis l’après guerre, de plusieurs micro-cultures instrumentales déployant des outils mathématiques, des objectifs et des modes opératoires différents. Toutefois, une certaine continuité épistémique traverse ces différentes cultures techniques et se matérialise dans des espaces d'échanges de savoirs théoriques, expérimen-

\footnotetext{
23 ACESR, «Nature des recherches poursuivies», s.d. [1964].

24 Ibid.

25 ACESR, Francis Cambou, «Projet de création d'un Institut d'études spatiales des rayonnements», 1964 (Annexe).

26 ACESR, Francis Cambou, «Éléments pour Monsieur le Recteur à propos du Centre d'étude spatiale des rayonnements», décembre 1968.
} 
taux et instrumentaux dont la rhétorique est commune aux cultures heuristiques de chacun des groupes de chercheurs en interaction. Ces trading zones, identifiées par Peter Galison dans le cas de la microphysique, permettent le déploiement d'un langage commun minimal pour des pratiques, des concepts et des résultats (Galison, I997, p. 803-844). Les instruments génériques de la physique nucléaire (comme les spectromètres ou les accélérateurs) constituent des objets circulant dans les différents sous-groupes et dont la ductilité expérimentale sert des objectifs scientifiques forts variés.

La physique spatiale développée au sein du CESR, en tant qu'elle constitue une pratique fondatrice de l'écologie toulousaine, emprunte donc, sur plusieurs de ses thématiques, aux cultures expérimentales communes de la physique nucléaire. Toutefois, nous l'avons vu, il est indispensable au petit groupe rassemblé autour de Francis Cambou de se distinguer et de se différencier du Laboratoire de physique nucléaire de Daniel Blanc. Les membres du CESR proposent donc un ensemble de recherches qui s'écartent, tant par les objets saisis, les méthodologies mobilisées que par les techniques mises en jeu, des thématiques de la physique nucléaire. Le territoire spatial constitue un bon moyen de pointer les différences avec la physique des particules. Dans le fascicule présentant, en 1964, le projet de «création d’un Institut d'études spatiales des rayonnements», Francis Cambou souligne que «penser et préparer une expérience spatiale oblige à des "réflexes" que les recherches habituelles ne nécessitent pas et entraînent d'énormes investissements». Les procédures de sélection des expériences sont davantage cadrées : les «satellites français [...] seront conçus, prévient Cambou, en fonction des expériences choisies par le comité scientifique et qui implique l'obligation de définir l'expérience deux ou trois ans à l'avance $\gg^{27}$. La temporalité du Laboratoire de physique spatiale n'est dès lors plus réductible à la temporalité des autres Laboratoires de physique nucléaire; les enjeux expérimentaux sont également hors des normes classiques. Cambou précise que «le but scientifique de l’opération étant défini, il

27 ACESR, Francis Cambou, «Projet de création d'un Institut d'études spatiales des rayonnements $\gg, 1964$. 
importe de grouper autour de celle-ci des chercheurs et des techniciens qui doivent pousser la minutie jusqu'aux limites les plus reculées $»^{28}$.

Le quatrième axe de recherche, présentée au recteur en 1968, porte sur les «phénomènes atmosphériques»; il est plus vague que les autres secteurs déjà cités et rassemble des observations sur les constituants de l'atmosphère. Les emprunts disciplinaires sont également plus variés et se détachent assez nettement de la physique nucléaire. L'équipe du CESR a développé un «chromatographe embarquable en ballon» pour effectuer des mesures «de vapeur d'eau de gaz carbonique ». L'instrument générique mobilisé est tiré du corpus technique de la chimie. De même, la recherche de la distribution verticale des composants de l'atmosphère à pour objectif d'apporter une «contribution importante à la connaissance météorologique des mouvements de circulation à grande échelle». À plus long terme, cette réflexion sur les modèles atmosphériques pourrait «s'étendre aux autres planètes $»^{29}$. Les mouvements disciplinaires esquissés ici tendent à s'éloigner de la physique nucléaire classique. Des prises extérieures sont évoquées, des lignes de fuite possibles sont tracées. On notera que Cambou, dans les rapports et les notes qu’il rédige entre 1964 et 1969 n'évoque qu'en passant les spécificités d’une recherche menée à l'aide de véhicules se déplaçant dans l'atmosphère ou au delà : il est probable que l'expérience accumulée est encore insuffisante pour tirer des conclusions épistémiques fortes sur les caractéristiques des observations spatiales liées aux collectes d'informations à distance ou aux biais introduits par les moyens de propulsions.

La physique spatiale toulousaine n’est pas encore constituée en écologie: les espaces éditoriaux disponibles pour rendre compte des expériences menées révèlent, au début des années 1960, une inconsistance thématique manifeste. Les publications du CESR pour l'année 1969 se répartissent entre des revues de géophysique (cinq articles dans les Annales de Géophysique, deux dans le Journal of Geophysical Research), des revues dédiées à la recherche instrumentales (trois publications dans Nuclear Instruments and Methods, un dans L'onde électrique, et un

28 Ibid.

29 ACESR, Francis Cambou, «Éléments pour Monsieur le Recteur à propos du Centre d'étude spatiale des rayonnements», décembre 1968. 
dans la Review of Scientific Instrument), deux articles dans les Comptes rendus à l'Académie des sciences, un seul dans une revue de physique nucléaire (Translations of Nuclear Science) et deux dans des revues généralistes orientées vers l'espace (Sciences et Industrie Spatiale, Aéronautique et Astronautique) ${ }^{30}$. Cet éclatement des publications témoigne - plus nettement que ne le suggère l'exposition des axes de recherche dans les rapports de Francis Cambou - d'une distance prise avec la physique nucléaire dans l’auditoire visé (même si son substrat épistémique reste un point d'ancrage), d'une prédominance des questions instrumentales et d'un rattachement faute de mieux aux travaux de la géophysique, discipline qui capte les nombreuses pratiques s'échelonnant de la lithosphère aux strates les plus élevées de l'atmosphère. La géophysique s’impose donc, dans la perspective syncrétique qu'elle offre, comme une discipline refuge pour les membres du CESR en quête de distinction.

La capacité du groupe à fonder en propre une écologie de physique spatiale à Toulouse se manifeste dans les instances de reproduction qu'il met en place. Dès 1965, une «chaire de physique spatiale» est créée par décret ministériel, ainsi qu'un DEA de Géophysique, «option physique spatiale»; en 1967 un certificat de «géophysique générale» complète l'édifice pédagogique ${ }^{31}$. Francis Cambou insiste sur le fait que la mise en place d' «enseignement de Physique Spatiale à la fois au niveau du second cycle et à celui de la spécialisation » est motivée par «le désir d'assurer un recrutement en jeunes chercheurs $»^{32}$. La compétition avec les autres sous-disciplines de la physique (et plus particulièrement avec la physique des particules) sourd ici dans cette revendication d'une filière pédagogique autonome. La rivalité disciplinaire est un moteur puissant de distinction pour une spécialité en plein essor (Edge et Mulkay, 1976, p. 256 ; Gilbert, 1977, p. I02). Dans le cas du CESR, elle est particulièrement vive puisqu'elle repose sur une homologie instrumentale quasi-parfaite.

30 ACESR, Rapport d'activité du CESR, 1968-1969, 15 septembre 1969.

31 ACESR, Centre d'étude spatiale des rayonnements, décembre 1968.

32 ACESR, Centre d'étude spatiale des rayonnements, novembre 1968. 
L'écologie de la physique spatiale en formation à Toulouse rassemble donc un petit groupe en fusion de chercheurs unis - au moins dans ce moment de transformation - par le souci d'un dépassement des structures préexistantes (i.e. la physique nucléaire condamnée à Toulouse faute d’un accélérateur, la décentralisation d'un certain nombre d'activités spatiales). La conversion à la physique spatiale passe par un réinvestissement technique et cognitif dont nous avons vu qu'il reposait sur une transposition d'instruments souches ainsi que sur la fidélité à une sous-culture instrumentale partagée par la physique nucléaire. Il s'agit donc d'une mutation opérée à moindre coût, mais dont les objectifs n'en reste pas moins un arrachement à l'ordre classique de la physique nucléaire. Le mouvement repéré dans les publications (en direction des auditoires spécialisées dans l'instrumentation et de ceux orientés vers la très généraliste géophysique) indique assez la détermination des physiciens toulousains à rompre avec leurs origines. La formation d'une écologie de la physique spatiale autour du CESR met en évidence des processus centrifuges de constitution d'un groupe fondés sur le maintien d'une culture physicienne et le rattachement à un corpus technique réinvesti dans un autre contexte que celui pour lequel il est prévu en physique nucléaire classique (i.e. transposer les spectromètres dans l'espace).

\section{PROJECTIONS ET JONCTIONS ESPÉRÉES}

Francis Cambou travaille sur un double front pour arrimer son écologie toulousaine à d'autres écologies. Nous l'avons vu, le laboratoire qu'il dirige est dans une position instable et fragile: Cambou reconnaît en décembre 1968 que «la recherche spatiale est aventureuse »33 : l'échec de certains projets ou les retards peuvent menacer l'existence du laboratoire. Sa quête d'alliances est motivée par la perspective d'une institutionnalisation plus définitive. Cambou mène donc, en direction de différentes écologies, locales et nationales, une campagne active dans les années 1964-1969 pour arrimer l'écologie de la physique spatiale à des écologies voisines susceptibles de lui fournir des appuis solides qui rendraient impossible sa disparition. Cambou précise d’abord les 
ancrages acquis : le «CESR est l'un des laboratoires sélectionnés par le CNES, et il est en outre associé au CNRS ». Le travail de jonction est d'abord une tentative d'autoreprésentation projetée et performative : Cambou propose, dans une note rédigée sous trois formes presque semblables, des montages institutionnels plaçant le CESR en position centrale. Le physicien met donc en place ce qu’Abbott désigne sous le nom d'avatar, c'est-à-dire toutes les entités d’approches projetés par les acteurs pour stabiliser une position en s'associant à d'autres écologies (Abbott, 2005, p. 265). Cambou construit des avatars de papier qui projette un laboratoire-idéal disposant d'une forte charge d'attraction et se déployant en un format organisationnel souple (et pour tout dire inédit).

En février 1969, Cambou rédige un «projet tendant à accorder au Centre d'étude spatiale des rayonnements le statut d'établissement à caractère scientifique et culturel». Son objectif est de «grouper, dans un Établissement pluridisciplinaire, des services de recherche fondamentale axés dans le domaine des sciences spatiales et des organismes divers dont les préoccupations sont liées au domaine spatial $»^{34}$. Jouant sur les échelles de territoires, Cambou explique que «le développement des sciences spatiales et de leur "retombées" dans le Complexe “Midi-Pyrénées" situe le présent projet à la fois dans le cadre national par sa finalité et dans le cadre régional par les incidences économiques et par l'environnement qu'il implique ». La perspective d'une Unité d'enseignement et de recherche (UER) fournit une forme possible pour l'institution projetée. L'UER de physique spatiale, créée en janvier 196935, avatar du CESR, est un double augmenté du laboratoire que dirige Cambou : elle rassemble le CESR, le Laboratoire de biologie médicale, qui s’intéresse «à l'action des rayonnements

34 ACESR, Francis Cambou, «Projet tendant à accorder au Centre d'étude spatiale des rayonnements le statut d'établissement à caractère scientifique et culturel», 5 février 1969. Les citations qui suivent sont toutes tirées de ce document. Un autre document, portant la même date, est intitulé «Projet concernant l'établissement à caractère scientifique et culturel pour le développement des sciences spatiales dans la région de Toulouse Midi-Pyrénées, et de leurs retombées». La rédaction du projet est légèrement différente (ajout d'adverbes) dans la deuxième version. Un troisième texte, non daté, s'intitule «Exposé des motifs pour la création d'un établissement destiné à promouvoir le développement des sciences spatiales en liaison avec les incidences industrielles et économiques». Une nouvelle fois les écarts de rédaction sont minimes.

35 ACESR, «Lettre du Recteur G. Richard au Doyen J. Blaizot», 8 janvier 1969. 
sur les cellules biologiques » et le Laboratoire de magnétisme et d'électronique quantique, mettant en œuvre «des procédés modernes de mesures magnétiques pour répondre aux besoins exprimés par les géophysiciens ». Les termes de Cambou sont sans ambiguïtés : il évoque une UER dont le CESR constituerait le pivot central, fonctionnant, sur un mode pluridisciplinaire, «dans le cadre des statuts d'un Établissement ayant sa propre gestion $»^{36}$. L'avatar ainsi décrit s'ancre dans une temporalité indécise: l'UER vient juste d'être créée, elle constitue une forme quasi-vide à laquelle Cambou offre les premières stries consistantes; mais il s'agit d’une prophétie que le directeur du CESR espère autoréalisatrice. Nous ne connaissons pas les destinataires du projet de Cambou, mais il n'est pas douteux que la description qu'il fait de l'UER a pour objectif de prendre de court ses concurrents potentiels. L'avatar de papier ainsi dessiné offre une projection cohérente du CESR; il gagne d’ailleurs en densité en dégageant des jonctions potentielles.

Les liens envisagés par Cambou s'organiseraient sur le mode d'une association intime avec «des organismes liés aux considérations économiques et aux applications de la haute technicité acquise lors du management spatial» ainsi que des «services industriels ou certains services de recherche appliquée liées aux grands Corps de l'État». La liste établie par Cambou est particulièrement copieuse et indique bien son souhait d'offrir une surface d’adhérence large : la Délégation à l’aménagement du territoire et à l'action régionale s'impose comme un acteur d'implantation locale, les «services d'obédience militaire ou nationale» (comme le Commissariat à l'énergie atomique ou le Centre de prospective des armées) seraient des clients potentiels d'un ensemble de connaissance «de l'environnement spatial», les «services industriels» (au titre desquels on trouve l'entreprise Motorola ou les établissement relevant de l’Union des sociétés industrielles aérospatiales et spatiales) envisageraient les techniques d’application, enfin, des «économistes qui souhaiteraient suivre de près l'évolution économique et industrielle d’une région» pourrait occuper l’axe réflexif du

36 ACESR, Francis Cambou, «Projet tendant à accorder au Centre d'étude spatiale des rayonnements le statut d'établissement à caractère scientifique et culturel», 5 février 1969. Les citations qui suivent sont tirées du même document. 
projet. Dans cet organigramme de liens multiples, l'UER occupe une place centrale : il en est «le pôle d'attraction intellectuel». Les écologies citées pour dégager une surface sociale large orientée sur les questions spatiales ne nécessitent pas forcément leur «coexistence géographique».

Cambou indique qu'il a établi cette première liste grâce à des «contacts personnels». Les jonctions qu'il entrevoit reposeraient essentiellement sur un «courant d'échanges et d'informations » qui circuleraient entre les différents acteurs. Cette perspective reste floue et les formes d'articulation ne sont pas même évoquées. Seule la philosophie générale de ces jonctions espérées est mentionnée: il s'agit, précise Cambou, de fournir à l'UER de physique spatiale une meilleur «facilité d’adaptation». La crainte de voir l'écologie mise en place détruite ou démantelée motive la sortie d'un isolement mortifère. Le directeur du CESR explique ainsi en préambule de son projet :

Il paraît hasardeux et même dangereux de cristalliser une communauté scientifique autour de projets qui pour des raisons de fluctuations économiques, politiques ou financières peuvent disparaître brutalement, même s'ils sont très engagées; l'exemple américain est très significatif à ce sujet et certains établissements de recherche spatiale connaissent en 1969 une crise très grave. Il apparaît nécessaire d'associer très intimement une telle communauté scientifique à des organismes liées aux considérations économiques et aux applications de la haute technicité.

Cette première ébauche noue à la fois un double «augmenté » du CESR, tête de pont d'un pôle scientifique orientée sur la recherche spatiale fondamentale et un ensemble d'écologies plus ou moins lointaines (géographiquement et thématiquement) capables de s'y associer. L’objectif est de créer un écosystème diversifié, capable de compenser ou d'amortir les probables retournements de tendance auquel le spatial balbutiant est sujet. Les jonctions envisagées sont encore très floues et reposent principalement sur un flux d'informations qui circulerait librement. La question de la synchronie des écologies est hautement spéculative: à l'intérieur même de l'avatar-UER, les laboratoires présentées n'ont pas tous le même degré de maturité (le Laboratoire de biologie médicale ne s'intéresse au spatial que de manière très périphérique). 


\section{CONCLUSION \\ Une topologie socio-épistémique du laboratoire}

Le processus écologique dans lequel est impliquée la physique spatiale toulousaine s'est donc d'abord centré autour d'un lieu conquis de haute lutte (en raison de la concurrence très forte sur le campus avec la physique nucléaire). Le mouvement épigénétique permettant la stabilisation du locus mêle des appuis scientifiques et politiques qui ne sont pas réductibles à une téléologie positive. La formation d'un groupe en fusion transformant un ensemble de pratiques inertes (i.e. la proximité avec le Centre technique du CNES, la décentralisation, l’obsolescence programmée de la physique nucléaire à Toulouse) définit - par agrégation de compétences et affirmation d'une légitimité épistémique concourante - un territoire de recherche partiellement autonome. Le détachement avec la physique nucléaire (matrice originelle du CESR) n'est pas total, mais il opère par une série de ruptures diachroniques: la maîtrise d'un corpus instrumental identique à celui de la physique nucléaire renforce la légitimité épistémique du petit groupe, mais les investissements éditoriaux sont encore désordonnés, même s'ils s'orientent globalement vers la géophysique. Enfin, le travail de jonction passe par la création d'un double du CESR : une UER centrée sur la physique spatiale. Les jonctions espérées sont découpées par Francis Cambou dans l'épaisseur des écologies voisines (thématiquement ou géographiquement) afin de conjurer un isolement qui pourrait condamner le laboratoire.

L'écologie physique spatiale reste, dans la deuxième moitié des années I960, en voie de constitution : elle n’a pas encore la robustesse suffisante pour occuper un territoire bibliographique cohérent, sa sécurité immobilière est à peine conquise et l'avatar écologique, en projection, témoigne assez des nécessités d’affermissement.

L'entrée en laboratoire de la physique spatiale repose sur une série très hétérogène de processus socio-épistémiques que l’analyse en termes d'écologie permet d'interpréter. La transformation des structures préexistantes ressortit à une chronologie aux multiples dimensions : le temps long de la décentralisation vient ici soutenir le temps court d’une conversion épistémique brutale. 
De même, les appuis mobilisés pour stabiliser le lieu engagent des rapports différents à la durée et à la taille des structures sociales mises en jeu : la massivité du Plan n’a que peu de choses à voir avec la bataille immobilière que mène Cambou. La constitution d'un laboratoire de physique spatiale relève, à la fin des années 1960, de mouvements épistémologiques profonds (l'émergence du spatial comme territoire scientifique nouveau), ainsi que d'arrangements immobiliers intenses (la conquête d'un lieu), d’ajustements professionnels et générationnels (la montée en puissance d’une jeune génération de chercheurs). La cristallisation imparfaite d'une écologie locale signale l'entremêlement des rapports de force que quintessencie la création d'un nouveau laboratoire.

En intégrant l'histoire d'une spécialité scientifique (i.e. la physique spatiale) à la théorie des écologies liées, il est possible de déplacer les analyses classiques de sociologie des sciences: il ne s'agit pas de soutenir la prééminence d'un facteur déterminant sur tous les autres, mais au contraire de comprendre comment les temporalités qui s'articulent pour chacun de ces facteurs (scientifiques, techniques, politiques, institutionnels) participent du processus d'émergence d'un laboratoire. L’autonomie relative des pratiques scientifiques (Bourdieu, 1975) est toujours maintenue au prix d'une tentative toujours renouvelée, par les dirigeants du laboratoire, de contrôler les processus politiques et institutionnels dans lesquels ils évoluent.

NdA. Je remercie Nicolas Adell, Emmanuel Barot, Jean-Louis Fabiani, Pascale Laborier, Frédéric Lebaron, Claude Rosental, Arnaud Saint-Martin, Simon Schaffer, Terry Shinn et Laurent Willemez pour leurs remarques et commentaires sur les versions antérieurs de ce texte. 


\section{BIBLIOGRAPHIE}

ABBOTT A., 2003, «Écologies liées: à propos du système des professions》, in Pierre-Michel Menger (dir.), Les Professions et leurs sociologies, Paris, Éditions de la Maison des sciences de l'homme, p.29-50.

-, 2005, «Linked Ecologies: States and Universities as Environments for Professions», Sociological Theory, 23-3, p. 245-274.

BEN-DAVID J. et COLLINS R., 1966, «Social Factors in the Origins of a New Science: The Case of Psychology », American Sociological Review, 34-4, p. 44I-465.

BIGG Ch., 200I, Behind the Lines. Spectroscopic Enterprises in Early Twentieth Europe, Thèse de doctorat en histoire, Université de Cambridge.

BOURDIEU P., 1975, «Les spécificités du champ scientifique et les conditions sociales du progrès de la raison», Sociologie et Sociétés, 7-I, p. 91-||8.

CONWAY E. M., 2006, «Drowning in Data: Satellite Oceanography and Information Overload in the Earth Sciences », Historical Studies in the Physical and Biological Sciences, 37-I, p. |27-I5I.

DEMAZIERE D. et JOUVENET M. (dir.), 2016, Andrew Abbott et l'héritage de l'école de Chicago, Paris, Éditions de l'EHESS.

EDGE D. O. et MULKAY M. J., 1976, Astronomy Transformed. The Emergence of Radio Astronomy in Britain, New York, John Wiley \& Sons.

FABIANI J.-L., 2003, «Pour en finir avec la réalité unilinéraire. Le parcours méthodologique de Andrew Abbott», Annales. Histoire, Sciences Sociales, 3, p. 549-465.

FORMAN P., 197I, «Weimar Culture, Causality, and Quantum Theory, 1918-1927: Adaptation by German Physicists and Mathematicians to a Hostile Intellectual Environment», Historical Studies in the Physical Sciences, 3, p. I- II5.

GALISON P., 1997, Image and Logic. A Material Culture of Microphysics, Chicago, The University of Chicago Press.

- et HEVLY B. W. (dir.), 1992, Big Science. The Growth of Large-Scale Research, Stanford, Stanford University Press, 1992.

GIERYN Th., 2002, «What Buildings Do », Theory and Society, 3I-I, p. 35-74. 
GILBERT N. G., 1977, «Competition, Differentiation and Careers in Science », Social Science Information, 16-I, p. 103-123.

HODDESON L., BRAUN E., TEICHMANN J. et WEART S., (dir.) 1992, Out of the Crystal Maze. Chapters from the History of Solid-State Physics, Oxford, Oxford University Press.

JOERGES B. et SHINN T. (dir), 200I, Instrumentation. Between Science, State and Industry, Dordrecht, Kluwer Academic Publisher.

KRIGE J., 2000, «Crossing the Interface from R\&D to Operational Use: the Case of the European Meteorological Satellite», Technology and Culture, 4I-I, p. 27-50.

LAMY J., 201 la, «What Did Management to Space Projects. The Franco-Soviet Project ARCAD 3 in the Late 1970s », Science in Context, 24-4, p. 545-586.

-, 20 I lb, «Grandeur scientifique et politiques de l'espace: la création et le transfert du CNES (1958-1974) », Revue d'histoire moderne et contemporaine, 58-I, p. I56-177.

- et DAVOUST E., 2009, «General-Purpose and Dedicated Regimes in the Use of Large Telescopes of the 20th Century», Journal of Astronomical History and Heritage, 12-3, p. 189-200.

MACK P. E., 1990, Viewing the Earth. The Social Construction of the Landsat Satellite System, Cambridge (Mass.), The MIT Press.

MULLINS N. C., 1972, «The Development of a Scientific Speciality: The Phage Group and the Origins of Molecular Biology», Minerva, 10, p. 51-82.

NAYLOR S., 2005, «Historical Geographies of Science: Places, Contexts, Cartographies», British Journal for the History of Science, 38-I, p. I-12.

PESTRE D., 2004, «Le nouvel univers des sciences et des techniques », in Amy Dahan et Dominique Pestre (dir.), Les Sciences pour la guerre (1940-1960), Paris, Éditions de l'EHESS, p. II-47.

SAINT-MARTIN A., 2008, L'Office et le télescope. Une sociologie historique de l'astronomie française, 1900-1940, Thèse de doctorat, Université Paris IV.

SARTRE J.-P., 1960, Critique de la raison dialectique. T.I, Théorie des ensembles pratiques, Paris, Gallimard, 1960. 
SHINN T., 1993, «The Bellevue Grand Électroaimant, 1900-1940: Birth of a ResearchTechnology Community», Historical Studies in the Physical Sciences, 24-I, p. I57-I87. -, 1998, «Change or Mutation? Reflections on the Foundations of Contemporary Science», Social Science Information, 38-I, p. 149-176.

- et RAGOUET P., 2005, Controverses sur la science. Pour une sociologie transversaliste de l'activité scientifique, Paris, Raisons d'agir.

STAR S. L. et GRIESEMER J. R., 1989, «Institutional Ecology, "Translations" and Boundary Objects: Amateurs and Professionals in Berkeley's Museum of Vertebrate Zoology, 1907-1939», Social Studies of Science, 19-3, p. 387-420.

WEBER Max, 1995 (1921), Économie et Société, T. I, Les Catégories de la sociologie, Paris, Pocket. 